\title{
WELL-POSEDNESS OF THE CAUCHY PROBLEM FOR SYSTEM OF OSCILLATORS ON 2D-LATTICE IN WEIGHTED $L^{2}$-SPACES
}

\begin{abstract}
S. M. Bak, G. M. Kovtonyuk. Well-posedness of the Cauchy problem for system of oscillators on 2D-lattice in weighted $l^{2}$-spaces, Mat. Stud. 56 (2021), 176-184.

We consider an infinite system of ordinary differential equations that describes the dynamics of an infinite system of linearly coupled nonlinear oscillators on a two dimensional integer-valued lattice. It is assumed that each oscillator interacts linearly with its four nearest neighbors and the oscillators are at the rest at infinity. We study the initial value problem (the Cauchy problem) for such system. This system naturally can be considered as an operator-differential equation in the Hilbert, or even Banach, spaces of sequences. We note that $l^{2}$ is the simplest choice of such spaces. With this choice of the configuration space, the phase space is $l^{2} \times l^{2}$, and the equation can be written in the Hamiltonian form with the Hamiltonian $H$. Recall that from a physical point of view the Hamiltonian represents the full energy of the system, i.e., the sum of kinetic and potential energy. Note that the Hamiltonian $H$ is a conserved quantity, i.e., for any solution of equation the Hamiltonian is constant. For this space, there are some results on the global solvability of the corresponding Cauchy problem. In the present paper, results on the $l^{2}$-well-posedness are extended to weighted $l^{2}$-spaces $l_{\Theta}^{2}$. We suppose that the weight $\Theta$ satisfies some regularity assumption. Under some assumptions for nonlinearity and coefficients of the equation, we prove that every solution of the Cauchy problem from $C^{2}\left((-T, T) ; l^{2}\right)$ belongs to $C^{2}\left((-T, T) ; l_{\Theta}^{2}\right)$. And we obtain the results on existence of a unique global solutions of the Cauchy problem for system of oscillators on a two-dimensional lattice in a wide class of weighted $l^{2}$-spaces. These results can be applied to discrete sine-Gordon type equations and discrete Klein-Gordon type equations on a two-dimensional lattice. In particular, the Cauchy problems for these equations are globally well-posed in every weighted $l^{2}$-space with a regular weight.
\end{abstract}

1. Introduction. Recently, considerable attention has been paid to models that are discrete in the spatial variables. Among the equations that describe such models, the most famous are the equations of chains of oscillators, the discrete sine-Gordon type equations, the FermiPasta-Ulam type systems and the discrete nonlinear Shrödinger type equations. Equations of such type are of interest in view of numerous applications in physics $[1,16,17]$.

Among the solutions of such equations, traveling waves deserve special attention. In papers $[3,15,19,20]$ traveling waves for infinite systems of linearly and nonlinearly coupled oscillators on 2D-lattice are studied. Papers [2, 6, 8] are devoted to the existence of homoclinic and heteroclinic traveling waves for the discrete sine-Gordon equations on 2D-lattice. The existence of solitary traveling waves in Fermi-Pasta-Ulam system on 2D-lattice is studied in $[10,12]$. A comprehensive presentation of existing results on traveling waves for 1D Fermi-Pasta-Ulam lattices is given in [22].

2010 Mathematics Subject Classification: 34A12, 34C15, 34G20.

Keywords: nonlinear oscillators; 2D-lattice; Cauchy problem; well-posedness; weighted sequence spaces. doi:10.30970/ms.56.2.176-184 
Papers $[1,4,21,26]$ deal with periodic in time solutions for such systems.

Another important class of solutions is standing waves. The existence of standing waves in discrete nonlinear Shrödinger type equations is studied in [11, 23, 24, 25].

In the present paper we study equations that describe the dynamics of an infinite system of linearly coupled nonlinear oscillators on a two dimensional lattice. Let $q_{n, m}=q_{n, m}(t)$ be a generalized coordinate of the $(n, m)$-th oscillator at time $t$. It is assumed that each oscillator interacts linearly with its four nearest neighbors. The equations of motion of the system are of the form

$$
\begin{gathered}
\ddot{q}_{n, m}=a_{n-1, m} q_{n-1, m}+a_{n, m} q_{n+1, m}+b_{n, m-1} q_{n, m-1}+b_{n, m} q_{n, m+1}+c_{n, m} q_{n, m}- \\
-V_{n, m}^{\prime}\left(q_{n, m}\right), \quad(n, m) \in \mathbb{Z}^{2},
\end{gathered}
$$

where $q_{n, m}$ is a sequence of real functions, the coefficients $a_{n, m}, b_{n, m}, c_{n, m}$ are sequences of real numbers, and the nonlinearity $V_{n, m} \in C^{1}(\mathbb{R} ; \mathbb{R})$ is the on-site potential. Equations (1) form an infinite system of ordinary differential equations.

We study the initial value problem (the Cauchy problem) for system (1) with initial conditions

$$
q_{n, m}(0)=q_{n, m}^{(0)}, \dot{q}_{n, m}(0)=q_{n, m}^{(1)},(n, m) \in \mathbb{Z}^{2},
$$

where $\left\{q_{n, m}^{(0)}\right\}$ and $\left\{q_{n, m}^{(1)}\right\}$ are given real sequences. System (1) naturally can be considered as an operator-differential equation, namelly

$$
\ddot{q}=A q-B(q)
$$

where

$$
(A q)_{n, m}=a_{n-1, m} q_{n-1, m}+a_{n, m} q_{n+1, m}+b_{n, m-1} q_{n, m-1}+b_{n, m} q_{n, m+1}+c_{n, m} q_{n, m}
$$

and the nonlinear operator $B$ is defined by

$$
(B(q))_{n, m}=V_{n, m}^{\prime}\left(q_{n, m}\right),
$$

in the Hilbert, or even Banach, space $E$ of sequences. Within this framework, initial conditions (2) become

$$
q(0)=q^{(0)}, \dot{q}(0)=q^{(1)},
$$

where $q^{(0)}$ and $q^{(1)}$ are given elements of the space $E$.

By definition, a solution of equation (3) is a twice continuously differentiable function of $t$ with values in $E$, that satisfies this equation. If for given initial data the solution of problem (3), (4) is defined on the whole number line, then it is called a global solution. Otherwise, it is called a local solution.

We note that the simplest choice of $E$ is the Hilbert space $l^{2}=l^{2}\left(\mathbb{Z}^{2}\right)$ of real two-sided square summable sequences $q=\left\{q_{n, m}\right\}$ with the scalar product

$$
\left(q^{(1)}, q^{(2)}\right)=\sum_{(n, m) \in \mathbb{Z}^{2}} q_{n, m}^{(1)} q_{n, m}^{(2)}
$$

and with the corresponding norm $\|q\|=(q, q)^{\frac{1}{2}}$. In particular, papers [5, 7, 9] are devoted to the well-posedness of initial value problem for infinite systems of linearly coupled nonlinear oscillators on 2D-lattice, while [13] and [14] are devoted to the well-posedness of initial value problem for such systems on 1D-lattice in $l^{2}$. 
2. Existence and uniqueness of global solutions in $l^{2}$. Throughout the paper we impose the following assumptions:

(i) $\left\{a_{n, m}\right\},\left\{b_{n, m}\right\}$ and $\left\{c_{n, m}\right\}$ are bounded;

(ii) $V_{n, m} \in C^{1}(\mathbb{R}), V_{n, m}(0)=V_{n, m}^{\prime}(0)=0,(n, m) \in \mathbb{Z}^{2}$, and $V_{n, m}^{\prime}$ is locally Lipschitz continuous uniformly with respect to $(n, m) \in \mathbb{Z}^{2}$, i.e., for any $R>0$ there exists a constant $C=C(R)>0$ such that for all $(n, m) \in \mathbb{Z}^{2}$

$$
\left|V_{n, m}^{\prime}\left(r_{1}\right)-V_{n, m}^{\prime}\left(r_{2}\right)\right| \leq C\left|r_{1}-r_{2}\right|,\left|r_{1}\right|,\left|r_{2}\right| \leq R .
$$

Sometimes we use the following stronger than (ii) assumption

$\left(i i^{\prime}\right)$ assumption $(i i)$ is satisfied with the constant $C>0$ independent of $R$, i.e., $V_{n, m}^{\prime}$ is globally Lipschitz continuous uniformly with respect to $(n, m) \in \mathbb{Z}^{2}$.

Assumption $(i)$ guaranties that the operator $A$ is bounded self-adjoint operator in $l^{2}$. With this choice of the configuration space, the phase space of equation (3) is $l^{2} \times l^{2}$, and the equation can be written in the Hamiltonian form

with the Hamiltonian

$$
\left\{\begin{array}{l}
\dot{p}=-H_{q}^{\prime}(p, q) \\
\dot{q}=H_{p}^{\prime}(p, q)
\end{array}\right.
$$

$$
H(p, q)=\frac{1}{2}\left(\|p\|^{2}-(A q, q)\right)+\sum_{(n, m) \in \mathbb{Z}^{2}} V_{n, m}\left(q_{n, m}\right),
$$

where $p=\dot{q}$. The Hamiltonian $H$ is a conserved quantity (see [7]), i.e., for any solution $q(t)$ of equation (3)

$$
H(\dot{q}(t), q(t))=H(\dot{q}(0), q(0))=H\left(q^{(1)}, q^{(0)}\right)=\text { Const } .
$$

Now we reproduce some results from $[5,7,9]$. The first one is a simple straightforward consequence of classical theorem on existence and uniqueness of global solutions for operator differential equations in Banach spaces.

Proposition 1 ([9], Theorem 4). Assume $(i)$ and $\left(i i^{\prime}\right)$. Then for every $q^{(0)} \in l^{2}$ and $q^{(1)} \in l^{2}$ problem (3), (4) has a unique global solution.

The proof of the next proposition makes use of Proposition 1, the Hamiltonian structure of the equation and cutt-off argument.

Proposition 2 ([7], Theorem 3). Assume (i) and (ii). Suppose that the operator $A$ is nonpositive, i.e., $(A q, q) \leq 0$ for all $q \in l^{2}$. Suppose also that one of the following two conditions holds:

(a) $V_{n, m}(r) \geq 0$ for all $(n, m) \in \mathbb{Z}^{2}$ and $r \in \mathbb{R}$;

(b) there exists a nondecreasing function $h(\xi), \xi \geq 0$, such that $\lim _{\xi \rightarrow+\infty} h(\xi)=+\infty$ and $V_{n, m}(r) \geq h(|r|)$ for all $(n, m) \in \mathbb{Z}^{2}$ and $r \in \mathbb{R}$.

Then for every $q^{(0)} \in l^{2}$ and $q^{(1)} \in l^{2}$ problem (3), (4) has a unique global solution.

A completely different type of nonlinearities is considered in the following proposition. 
Proposition 3 ([5], Corollary 3.1). Suppose that condition $(i)$ is satisfied, $V_{n, m}(r)=\frac{g_{n, m}}{p} r^{p}$, $p>2$, where $\left\{g_{n, m}\right\}$ is a bounded sequence, and the operator $A$ is negative definite, i.e., $(A q, q) \leq-\alpha\|q\|^{2}$, where $\alpha>0$. Then there exists $\delta>0$ such that for any $q^{(0)}, q^{(1)} \in l^{2}$ with $\left\|q^{(0)}\right\| \leq \delta$ and $\left\|q^{(1)}\right\| \leq \delta$, problem (3), (4) has a unique global solution.

3. Existence and uniqueness of global solutions in weighted spaces. The aim of the present paper is to extend the $l^{2}$-well-posedness results to weighted $l^{2}$-spaces and, hence, provide a refined information about problem $(1),(2)$.

Let $\Theta=\left\{\theta_{n, m}\right\}$ be a sequence of positive numbers (weight). We denote by $l_{\Theta}^{2}$ the space of all two-sided sequences $q=\left\{q_{n, m}\right\}$ of real numbers such that the norm

$$
\|q\|_{\Theta}=\left(\sum_{(n, m) \in \mathbb{Z}^{2}} \theta_{n, m}\left|q_{n, m}\right|^{2}\right)^{\frac{1}{2}}
$$

is finite. This is a Hilbert space with the scalar product

$$
\left(q^{(1)}, q^{(2)}\right)_{\Theta}=\sum_{(n, m) \in \mathbb{Z}^{2}} \theta_{n, m} q_{n, m}^{(1)} q_{n, m}^{(2)} .
$$

We suppose that the weight $\Theta=\left\{\theta_{n, m}\right\}$ satisfies the following assumption:

(iii) the weight $\Theta$ is a regular, i.e., the sequence $\left\{\theta_{n, m}\right\}$ is bounded below by a positive constant and there exists a constant $c_{0}>0$ such that

for all $(n, m) \in \mathbb{Z}^{2}$.

$$
c_{0}^{-1} \leq \frac{\theta_{n+1, m}}{\theta_{n, m}}, \frac{\theta_{n, m+1}}{\theta_{n, m}} \leq c_{0}
$$

Under this assumption weighted space $l_{\Theta}^{2}$ is densely and continuously embedded into $l^{2}$, and

$$
\|q\| \leq C_{1}\|q\|_{\Theta}, q \in l_{\Theta}^{2}
$$

with some $C_{1}>0$. Therefore, all these spaces are densely and continuously embedded into the space $l^{\infty}$ of bounded sequences with norm

$$
\|q\|_{l^{\infty}}=\sup _{(n, m) \in \mathbb{Z}^{2}}\left|q_{n, m}\right| .
$$

And if $\theta_{n, m} \equiv 1$, then $l_{\Theta}^{2}=l^{2}$.

From the point of view of functional analysis assumption ( $i i i)$ is quite natural. It means that the space $l_{\Theta}^{2}$ is translation invariant. More precisely, let $T_{+}^{(i)}$ and $T_{-}^{(i)}(i=1,2)$ be the operators of right and left shifts, respectively, defined by

$$
\begin{aligned}
& \left(T_{+}^{(1)} q\right)_{n, m}=q_{n-1, m},\left(T_{-}^{(1)} q\right)_{n, m}=q_{n+1, m}, \\
& \left(T_{+}^{(2)} q\right)_{n, m}=q_{n, m-1},\left(T_{-}^{(2)} q\right)_{n, m}=q_{n, m+1} .
\end{aligned}
$$

Lemma 1. Assumption (iii) holds if and only if all operators $T_{ \pm}^{(k)}(k=1,2)$ are bounded in $l_{\Theta}^{2}$.

Proof. Indeed, we have that

$$
\left\|T_{+}^{(1)} q\right\|_{\Theta}^{2}=\sum_{(n, m) \in \mathbb{Z}^{2}} \theta_{n, m}\left|q_{n-1, m}\right|^{2}=\sum_{(n, m) \in \mathbb{Z}^{2}} \theta_{n+1, m}\left|q_{n, m}\right|^{2}=\sum_{(n, m) \in \mathbb{Z}^{2}} \theta_{n, m} \frac{\theta_{n+1, m}}{\theta_{n, m}}\left|q_{n, m}\right|^{2},
$$


which means that $T_{+}^{(1)}$ is bounded in $l_{\Theta}^{2}$ if and only if $\frac{\theta_{n+1, m}}{\theta_{n, m}}$ is bounded.

Similarly, $T_{-}^{(1)}, T_{+}^{(2)}, T_{-}^{(2)}$ are bounded in $l_{\Theta}^{2}$ if and only if $\frac{\theta_{n-1, m}}{\theta_{n, m}}, \frac{\theta_{n, m+1}}{\theta_{n, m}}, \frac{\theta_{n, m-1}}{\theta_{n, m}}$ are bounded.

The most important examples of weights satisfying assumption (iii) are power and exponential weights:

$$
\theta_{n, m}=(1+|n|+|m|)^{\alpha}, \alpha>0
$$

and

$$
\theta_{n, m}=\exp (\alpha(|n|+|m|)), \alpha>0 .
$$

Lemma 2. Assume $(i)$ and (iii). Then the operator $A$ is bounded in $l_{\Theta}^{2}$.

Proof. The operator $A$ can be represented in the form

$$
A=a \circ T_{-}^{(1)}+T_{+}^{(1)} \circ a+b \circ T_{-}^{(2)}+T_{+}^{(2)} \circ b+c,
$$

where $a, b$ and $c$ are the operators of multiplication by the sequences $\left\{a_{n, m}\right\},\left\{b_{n, m}\right\}$ and $\left\{c_{n, m}\right\}$ respectively, and $\circ$ stands for the composition of operators. Since the operators $T_{-}^{(i)}$, $T^{(i)}+(i=1,2), a, b$ and $c$ are bounded operators in $l_{\Theta}^{2}$ by Lemma 1 and assumption $(i)$ respectively, we conclude that $A$ is bounded in $l_{\Theta}^{2}$.

Lemma 3. Under assumption ( $i i)$, the nonlinear operator $B$ is a locally Lipschitz continuous operator in the space $l_{\Theta}^{2}$, i.e., for any $R>0$ there exists a constant $C=C(R)>0$ such that

$$
\left\|B\left(q^{(1)}\right)-B\left(q^{(2)}\right)\right\|_{\Theta} \leq C\left\|q^{(1)}-q^{(2)}\right\|_{\Theta}
$$

for all $q^{(i)} \in l_{\Theta}^{2}$ with $\left\|q^{(i)}\right\|_{\Theta} \leq R(i=1,2)$. If assumption $\left(i i^{\prime}\right)$ is satisfied, then the operator $B$ is globally Lipschitz continuous operator in the space $l_{\Theta}^{2}$, i.e., the constant $C$ in inequality (6) can be chosen independent of $R$.

Proof. Let assumption (ii) be satisfied, $q \in l_{\Theta}^{2}$ and $\|q\|_{l_{\Theta}^{2}} \leq R$. Since

$$
\|q\|_{l^{\infty}} \leq\|q\| \leq C_{1}\|q\|_{\Theta} \leq C_{1} R=R_{1},
$$

inequality (5) and condition $V_{n, m}^{\prime}(0)=0$ mean that $\left|V_{n, m}^{\prime}\left(q_{n, m}\right)\right| \leq C\left|q_{n, m}\right|$ and

$$
\|B(q)\|_{\Theta}=\left(\sum_{(n, m) \in \mathbb{Z}^{2}} \theta_{n, m}\left|V_{n, m}^{\prime}\left(q_{n, m}\right)\right|^{2}\right)^{\frac{1}{2}} \leq C\left(\sum_{(n, m) \in \mathbb{Z}^{2}} \theta_{n, m}\left|q_{n, m}\right|^{2}\right)^{\frac{1}{2}}=C\|q\|_{\Theta} .
$$

Thus, $B(q) \in l_{\Theta}^{2}$ and $\|B(q)\|_{\Theta} \leq C\|q\|_{\Theta}$.

Now let $q^{(i)}=\left\{q_{n, m}^{(i)}\right\} \in l_{\Theta}^{2}$ and $\left\|q^{(i)}\right\|_{\Theta} \leq R(i=1,2)$. Then $\left\|q^{(i)}\right\|_{l^{\infty}} \leq R_{1}$ and (5) implies that

$$
\left|V_{n, m}^{\prime}\left(q_{n, m}^{(1)}\right)-V_{n, m}^{\prime}\left(q_{n, m}^{(2)}\right)\right| \leq C\left|q_{n, m}^{(1)}-q_{n, m}^{(2)}\right| .
$$

Similarly to the previous one, we obtain

$$
\begin{gathered}
\left\|B\left(q^{(1)}\right)-B\left(q^{(2)}\right)\right\|_{\Theta}=\left(\sum_{(n, m) \in \mathbb{Z}^{2}} \theta_{n, m}\left|V_{n, m}^{\prime}\left(q_{n, m}^{(1)}\right)-V_{n, m}^{\prime}\left(q_{n, m}^{(2)}\right)\right|^{2}\right)^{\frac{1}{2}} \leq \\
\leq C\left(\sum_{(n, m) \in \mathbb{Z}^{2}} \theta_{n, m}\left|q_{n, m}^{(1)}-q_{n, m}^{(2)}\right|^{2}\right)^{\frac{1}{2}}=C\left\|q^{(1)}-q^{(2)}\right\|_{\Theta} .
\end{gathered}
$$

Hence, $B$ is a locally Lipschitz continuous operator in the space $l_{\Theta}^{2}$.

The proof in the case $\left(i i^{\prime}\right)$ is similar. 
Lemma 4. Assume (i), (ii) and (iii). Suppose that $q \in C^{2}\left((-T, T) ; l^{2}\right)$ is a solution of problem (1), (2) with $q^{(0)}=\left\{q_{n, m}^{(0)}\right\} \in l_{\Theta}^{2}$ and $q^{(1)}=\left\{q_{n, m}^{(1)}\right\} \in l_{\Theta}^{2}$. Then $q \in C^{2}\left((-T, T) ; l_{\Theta}^{2}\right)$.

Proof. Let $q \in C^{2}\left((-T, T) ; l^{2}\right)$ be a solution of problem $(1),(2)$ with $q^{(0)}=\left\{q_{n, m}^{(0)}\right\} \in l_{\Theta}^{2}$ and $q^{(1)}=\left\{q_{n, m}^{(1)}\right\} \in l_{\Theta}^{2}$. Fix any $\tau \in(0, T)$ and set $R_{\tau}=\sup _{t \in[-\tau, \tau]}\|q(t)\|$. Let

$$
\tilde{V}_{n, m}^{\prime}(r)=\left\{\begin{array}{l}
V_{n, m}^{\prime}(r) \text { if }|r| \leq R_{\tau}+1, \\
\tilde{V}_{n, m}^{\prime}(r)=V_{n, m}^{\prime}\left(R_{\tau}+1\right) \text { if } r>R_{\tau}+1, \\
V_{n, m}^{\prime}\left(-R_{\tau}-1\right) \text { if } r<-R_{\tau}-1 .
\end{array}\right.
$$

Then on $[-\tau, \tau]$ the function $q(t)$ obviously solves the system

$$
\begin{gathered}
\ddot{q}_{n, m}=a_{n-1, m} q_{n-1, m}+a_{n, m} q_{n+1, m}+b_{n, m-1} q_{n, m-1}+b_{n, m} q_{n, m+1}+c_{n, m} q_{n, m}- \\
-\tilde{V}_{n, m}^{\prime}\left(q_{n, m}\right),(n, m) \in \mathbb{Z}^{2},
\end{gathered}
$$

with the same initial data.

Obviously, the functions $\tilde{V}_{n, m}^{\prime}$ satisfy assumption $\left(i i^{\prime}\right)$, and, by Lemma 3 , the corresponding operator $\tilde{B}$ is globally Lipschitz continuous operator in the space $l_{\Theta}^{2}$. By Lemma 2 , the linear operator $A$ is a bounded operator in the space $l_{\Theta}^{2}$. By the classical result ([18], Chapter 6 , Theorem 1.2), problem (7), (2) has a unique solution

$$
\tilde{q} \in C^{2}\left((-T, T) ; l_{\Theta}^{2}\right) \subset C^{2}\left((-T, T) ; l^{2}\right) .
$$

By uniqueness for the initial value problem in the space $l^{2}$, we have that $\tilde{q}=q$ on $[-\tau, \tau]$. Since $\tau \in(0, T)$ is an arbitrary point, we obtain that $q \in C^{2}\left((-T, T) ; l_{\Theta}^{2}\right)$.

Combining Lemma 4 with Propositions $1-3$, we obtain the following results.

Theorem 1. Assume $(i),\left(i i^{\prime}\right)$ and $(i i i)$. Then for every $q^{(0)} \in l_{\Theta}^{2}$ and $q^{(1)} \in l_{\Theta}^{2}$ problem (1), (2) has a unique global solution $q \in C^{2}\left(\mathbb{R} ; l_{\Theta}^{2}\right)$.

Theorem 2. Assume (i), (ii) and (iii). Suppose that the operator $A$ is non-positive, i.e., $(A q, q) \leq 0$ for all $q \in l^{2}$. Suppose also that one of the following two conditions holds:

(a) $V_{n, m}(r) \geq 0$ for all $(n, m) \in \mathbb{Z}^{2}$ and $r \in \mathbb{R}$;

(b) there exists a nondecreasing function $h(\xi), \xi \geq 0$, such that $\lim _{\xi \rightarrow+\infty} h(\xi)=+\infty$ and $V_{n, m}(r) \geq h(|r|)$ for all $(n, m) \in \mathbb{Z}^{2}$ and $r \in \mathbb{R}$.

Then for every $q^{(0)} \in l_{\Theta}^{2}$ and $q^{(1)} \in l_{\Theta}^{2}$ problem (1), (2) has a unique global solution $q \in$ $C^{2}\left(\mathbb{R} ; l_{\Theta}^{2}\right)$.

Theorem 3. Assume $(i)$ and (iii). Suppose that $V_{n, m}(r)=\frac{g_{n, m}}{p} r^{p}, p>2$, where $\left\{g_{n, m}\right\}$ is a bounded sequence, and the operator $A$ is negative definite in $l^{2}$. Then there exists $\delta>0$ such that for any $q^{(0)} \in l_{\Theta}^{2}$ and $q^{(1)} \in l_{\Theta}^{2}$ with $\left\|q^{(0)}\right\| \leq \delta$ and $\left\|q^{(1)}\right\| \leq \delta$, problem (1), (2) has a unique global solution $q \in C^{2}\left(\mathbb{R} ; l_{\Theta}^{2}\right)$.

Let us highlight that in Theorem 3 the smallness of the initial data with respect to $l^{2}$-norm, not in the space $l_{\Theta}^{2}$.

Now we present some examples that often appear in applications. 
Example 1. Taking

$$
(A q)_{n, m}=a\left(q_{n+1, m}+q_{n-1, m}+q_{n, m+1}+q_{n, m-1}-4 q_{n, m}\right)
$$

and

$$
V_{n, m}(r)=1-\cos r,
$$

one obtains the discrete sine-Gordon equation on a 2-dimensional lattice:

$$
\ddot{q}_{n, m}=a(\Delta q)_{n, m}-\sin q_{n, m},(n, m) \in \mathbb{Z}^{2},
$$

where $a>0$ and

$$
(\Delta q)_{n, m}:=q_{n+1, m}+q_{n-1, m}+q_{n, m+1}+q_{n, m-1}-4 q_{n, m}
$$

is the 2-dimensional discrete Laplacian.

In this case the nonlinearity satisfies $\left(i i^{\prime}\right)$. Hence, Theorem 1 shows that the Cauchy problem (8), (2) is globally well-posed in every space $l_{\Theta}^{2}$ with a regular weight $\Theta$.

Example 2. When

$$
(A q)_{n, m}=a(\Delta q)_{n, m}-k^{2} q_{n, m}, a>0
$$

and

$$
V_{n, m}(r)=-\frac{\theta}{4} r^{4}, \theta= \pm 1,
$$

we obtain the discrete nonlinear Klein-Gordon equation on a 2-dimensional lattice in the case when $k^{2}>0$ :

$$
\ddot{q}_{n, m}=a(\Delta q)_{n, m}-k^{2} q_{n, m}+\theta q_{n, m}^{3},(n, m) \in \mathbb{Z}^{2},
$$

and discrete nonlinear wave equation on a 2-dimensional lattice in the case when $k^{2}=0$ :

$$
\ddot{q}_{n, m}=a(\Delta q)_{n, m}+\theta q_{n, m}^{3},(n, m) \in \mathbb{Z}^{2},
$$

It is easy to verify that

$$
(\Delta q, q)=-\sum_{(n, m) \in \mathbb{Z}^{2}}\left(\left(q_{n, m}-q_{n-1, m}\right)^{2}+\left(q_{n, m}-q_{n, m-1}\right)^{2}\right)
$$

and, hence, the operator $\Delta$ is non-positive.

By Theorem 2, in the case when $\theta=1$, the potential $V_{n, m}=\frac{r^{4}}{4} \geq 0$ and the Cauchy problems (9), (2) and (10), (2) are globally well-posed in every space $l_{\Theta}^{2}$ with a regular weight $\Theta$.

On the other hand, in the case when $\theta=-1$, the potential $V_{n, m}=-\frac{r^{4}}{4} \leq 0$ and the operator $a \Delta-k^{2}$ is negative definite, and Theorem 3 guaranties the existence of unique global solution of the problem (9), (2) for all initial data in $l_{\Theta}^{2}$ that have sufficiently small $l^{2}$-norm, provided the weight $\Theta$ is regular. The case of the problem (10), (2) remains open. 


\section{REFERENCES}

1. S. Aubry, Breathers in nonlinear lattices: Existence, linear stability and quantization, Physica D, 103 (1997), 201-250.

2. S.M. Bak, Existence of heteroclinic traveling waves in a system of oscillators on a two-dimensional lattice, Matematychni Metody ta Fizyko-Mekhanichni Polya, 57 (2014), №3, 45-52. (in Ukrainian) (Engl. transl.: J. Math. Sci., 217 (2016), №2, 187-197.) doi:10.1007/s10958-016-2966-z

3. S.M. Bak, Existence of the solitary traveling waves for a system of nonlinearly coupled oscillators on the 2d-lattice, Ukr. Mat. Zh., 69 (2017), №4, 435-444. (in Ukrainian) (Engl. transl.: Ukr. Mat. J., 69 (2017), №4, 509-520.) doi:10.1007/s11253-017-1378-7

4. S.M. Bak, Existence of the time periodic solutions of system of oscillators on 2D-lattice, Carpathian Math. Publ., 4 (2012), №2, 5-12. (in Ukrainian)

5. S.M. Bak, Global well-posedness of the Cauchy problem for system of oscillators on 2D-lattice with power potentials, J. Math. Sci., 246 (2020), №5, 593-601. doi:10.1007/s10958-020-04765-6

6. S.M. Bak, Homoclinic traveling waves in discrete sine-Gordon equation with nonlinear interaction on 2D lattice, Mat. Stud., 52 (2019), №2, 176-184. doi:10.30970/ms.52.2.176-184

7. S.M. Bak, The existence and uniqueness of the global solution of the Cauchy problem for an infinite system of nonlinear oscillators on a two-dimensional lattice, Math. and Comp. Modelling. Ser.: Phys. and Math. Sci., 5 (2011), 3-9. (in Ukrainian)

8. S. Bak, The existence of heteroclinic traveling waves in the discrete sine-Gordon equation with nonlinear interaction on a 2D-lattice, J. Math. Phys., Anal., Geom., 14 (2018), №1, 16-26. doi:10.15407/mag14.01.016

9. S.M. Bak, O.O. Baranova, Yu.P. Bilyk, Correctness of the Cauchy problem for an infinite system of nonlinear oscillators on 2D-lattice, Math. and Comp. Modelling. Ser.: Phys. and Math. Sci., 4 (2010), 18-24. (in Ukrainian)

10. S.M. Bak, G.M. Kovtonyuk, Existence of solitary traveling waves in Fermi-Pasta-Ulam system on $2 D$ lattice, Mat. Stud., 50 (2018), №1, 75-87. doi:10.15330/ms.50.1.75-87

11. S. Bak, G. Kovtonyuk, Existence of standing waves in DNLS with saturable nonlinearity on $2 D$ lattice, Commun. Math. Analysis, 22 (2019), №2, 18-34.

12. S.M. Bak, G.M. Kovtonyuk, Existence of traveling waves in Fermi-Pasta-Ulam type systems on 2Dlattice, J. Math. Sci., 252 (2021), №4, 453-462. doi:10.1007/s10958-020-05173-6

13. S. Bak, G.M. N'Guerekata, A. Pankov, Well-posedness of initial value problem for discrete nonlinear wave equations, Commun. Math. Analysis, 8 (2010), №1, 79-86.

14. S.N. Bak, A.A. Pankov, On the dynamical equations of a system of linearly coupled nonlinear oscillators, Ukr. Math. J., 58 (2006), №6, 815-822. doi:10.1007/s11253-006-0105-6

15. S.N. Bak, A.A. Pankov, Traveling waves in systems of oscillators on 2D-lattices, J. Math. Sci., 174 (2011), №4, 916-920. doi:10.1007/s10958-011-0310-1

16. O.M. Braun, Y.S. Kivshar, Nonlinear dynamics of the Frenkel-Kontorova model, Physics Repts, 306 (1998), 1-108.

17. O.M. Braun, Y.S. Kivshar, The Frenkel-Kontorova Model, Concepts, Methods and Applications, Springer, Berlin, 2004.

18. Yu.L. Daleckii, M.G. Krein, Stability of solutions of differential equations in Banach spaces, Amer. Math. Soc., Providence, R. I., 1974.

19. M. Fečkan, V. Rothos, Traveling waves in Hamiltonian systems on 2D lattices with nearest neighbour interactions, Nonlinearity, 20 (2007), 319-341.

20. G. Friesecke, K. Matthies, Geometric solitary waves in a 2D math-spring lattice, Discrete and continuous dynamical systems, 3 (2003), №1, 105-114. 
21. R.S. MacKay, S. Aubry, Proof of existence of breathers for time-reversible a Hamiltonian networks of weakly coupled oscillators, Nonlinearity, 7 (1994), 1623-1643.

22. A. Pankov, Traveling Waves and Periodic Oscillations in Fermi-Pasta-Ulam Lattices, Imperial College Press, London-Singapore, 2005.

23. A. Pankov, Gap solitons in periodic discrete NLS equations, Nonlinearity, 19 (2006), 27-40.

24. A. Pankov, Gap solitons in periodic discrete nonlinear Schrödinger equations, II: generalized Nehari manifold approach, Discr. Cont. Dyn. Sys., 19 (2007), №2, 419-430.

25. A. Pankov, V. Rothos, V.Periodic and decaying solutions in DNLS with saturable nonlinearity, Proc. Roy. Soc. Sec. A., 464 (2008), 3219-3236.

26. P. Srikanth, On periodic motions of two-dimentional lattices, Functional analysis with current applications in science, technology and industry, 377 (1998), 118-122.

Vinnytsia Mykhailo Kotsiubynskyi State Pedagogical University

Vinnytsia, Ukraine

sergiy.bak@gmail.com, galyna.kovtonyuk@gmail.com

Received 11.02.2021

Revised 06.12.2021 